\title{
La Calidad de la Educación Universitaria. Peculiaridades del Modelo a Distancia
}

\author{
(Quality of the Education at University. \\ Peculiarities of Distance Model) \\ RAMón PÉREz JUSTE
Universidad Nacional de Educación a Distancia \\ (España)
}

\begin{abstract}
RESUMEN: El autor aboga por una concepción de la calidad de la educación universitaria capaz de integrar de modo armónico la calidad misma de los proyectos educativos y la de su gestión. Desde la primera perspectiva, defiende la necesidad de superar la tradicional autolimitación de la universidad a la simple transmisión del saber; en relación con la segunda, acude al modelo europeo de gestión de la calidad. Del mismo modo, el autor pone de relieve la necesidad de abordar la calidad a diferentes niveles, de forma que, para cada uno de ellos, se puedan establecer tanto las aportaciones que se esperan cuanto las responsabilidades que deberian asumir. Los planteamientos generales sobre calidad van acompañadas de reflexiones sobre su acomodación a las peculiaridades de la educación universitaria a distancia.
\end{abstract}

\section{Calidad - Evaluación - Formación}

ABSTRACT: The author advocates for a conception of the quality of the higher education that harmoniously integrates the quality of the educational projects "per se" and the quality of its management. From the first perspective, he defends the need of surpassing the traditional self-limitation of the university just to knowledge transmission; regarding the second perspective, he turns to the European quality management model. Likewise, the author emphasises the need of approaching the quality from different levels, so that it would be possible to establish, for each level, the expected contributions as well as the responsibilities to be assumed. The general positions on quality are accompanied of reflections on its accommodation to the peculiarities of the higher distance education.

\section{Quality - Evaluation - Training}




\section{INTRODUCCIÓN}

De un tiempo a esta parte, la calidad se ha convertido en un tema recurrente, tanto a nivel de conversación como de reflexión científica, de actuaciones prácticas, de desarrollos técnicos y tecnológicos y hasta de investigación aplicada.

Diversas son las razones que parecen explicar este hecho. Si en el marco de las empresas productoras de bienes de consumo y de las que ofrecen servicios a la sociedad hay una base de competencia y supervivencia, en el fondo puede subyacer esa importante necesidad de los seres humanos de superarse a sí mismos, de ir más allá, de avanzar, de ampliar el ámbito de sus necesidades, como afirmaba el profesor M. Yela al hablar de las necesidades básicas de la persona.

Desde luego, en el caso de la Educación entendida como servicio, parecen darse ambos tipos de razones. En efecto, si bien es cierto que los teóricos de la Pedagogía siempre que han escrito sobre Educación lo han hecho pensando en una educación de calidad, no lo es menos que los planteamientos sistemáticos sobre la calidad ocurren en un momento como el actual en que, de una parte, y debido a las circunstancias sociales1, aparece el factor de competencia entre instituciones, y, de otra, las demandas puramente cuantitativas -más escuelas y universidades, más recursos, mas profesores- ya han sido razonablemente satisfechas, surgiendo, en consecuencia, necesidades y demandas de carácter cualitativo,

Sea como fuere, el hecho es que, en nuestro país, y en los principales países del ámbito occidental -EE.UU., Japón, Unión Europea- se vienen desarrollando una serie de iniciativas orientadas en la dirección de la calidad que merecen ser tomadas en consideración y, probablemente, hasta participar en ellas como forma de mejorar nuestro sistema educativo.

Destacaremos entre ellas las convocatorias anuales de los premios a la calidad Deming y Malcolm Baldridge y, más recientemente, de la European Foundation for Quality Management (EFQM), el Proyecto Piloto para la evaluación de la calidad de las universidades o las dos convocatorias del Plan Nacional para la Evaluación de la Calidad de las Universidades, sin olvidar los numerosos Seminarios, Congresos e iniciativas de todo tipo en esta dirección.

(1) Entre otras, la exigencia de rendición de cuentas dadas las cuantiosas inversiones que conllevan los sistemas educativos o, en un orden muy diferente, la reducción de la natalidad... 


\section{EL MARCO CONFUSO DE LA CALIDAD}

Pocos conceptos resultan, a la vez, tan asequibles para su aprehensión intuitiva y $\tan$ difíciles para su definición rigurosa.

Varias son las razones que contribuyen a este hecho, entre las que podemos destacar las siguientes:

a) Los reduccionismos que se dan en su definición. Hay, en efecto, concepciones ligadas sólo a los productos o resultados (eficacia), a los procesos, solos o en unión de los productos (eficiencia), o a la naturaleza de los productos, bienes o servicios que se espera sean de calidad (especificaciones).

A ello hay que añadir los reduccionismos que se dan dentro de cada uno de tales bloques. El caso más evidente es la limitación de los productos a los de carácter económico o, en el ámbito educativo, a resultados puramente cognitivos, académicos, sin atención a las demás dimensiones del ser humano.

b) En el caso de la Educación, las diferentes concepciones filosóficas e ideológicas que subyacen a los planteamientos educativos. Este hecho resulta de especial transcendencia en la medida en que, como parece obvio, arrastra tras de sí las tomas de posición sobre los medios adecuados -estrategias, organización...- para el logro de los objetivos, además de reflejarse en lo que se consideran productos de la acción educativa.

\subsection{NATURALEZA DE LA CALIDAD}

El concepto de calidad es complejo y presenta manifestaciones diferentes que pueden ser entendidas como complementarias. Veamos las más importantes:

- La excelencia en las notas o características del objeto o servicio. De alguna forma, esta nota hace relación a un concepto de calidad que tiene que ver con las especificaciones de los servicios/productos de que se trate.

D El logro efectivo de un servicio/objeto excelente desde perspectivas objetivas. Puede apreciarse a través de la comparación entre especificaciones y realidades.

D La percepción de los objetos/servicios como algo más o menos satisfactorio. La satisfacción, en definitiva, de las necesidades, demandas y expectativas de los usuarios ${ }^{2}$, destinatarios o clientes.

(2) Th. H. Berry añade el término razonables a tales necesidades y expectativas. Vid. BERRY, Th.H. (1992): Cómo gerenciar la transformación hacia la Calidad Total, Santa Fé de Bogotá: McGraw Hill. 
- El logro de la excelencia, por medio de procesos eficientes que conducen a resultados eficaces. Los posibles costos asignables a la mejora de la calidad deben quedar, y pueden quedar de hecho, claramente compensados no ya por la reducción de los costos de la no calidad sino por la disminución de los costos derivada de una gestión eficiente ${ }^{3}$.

La mejora continua de la calidad es una característica de los sistemas de calidad, siempre posible porque cualquier proceso puede realizarse "más eficazmente y más eficientemente con menos derroche y consumo de recursos..." (Norma UNE 66 904-4)4.

- Un concepto más profundo de calidad es el que la entiende como algo global y unificador, "que englobe todo lo referente al objetivo de 'excelencia' al que debe tender toda empresa"s.

Al logro de estas manifestaciones contribuyen, de forma clara y decisiva, algunas notas o exigencias propias del sistema de calidad, entre las que se deben destacar las siguientes:

- La implicación de todo el personal, con los elementos añadidos de reconocimiento, motivación, formación, promoción, sentido de pertenencia..., en definitiva, por medio de la satisfacción en el puesto de trabajo.

- La extensión a todos los elementos, tanto aquellos que pueden considerarse críticos para la producción del bien o servicio cuanto a aquellos otros que facilitan la eficacia y la eficiencia en su producción.

- Su aplicación a todos los momentos del proceso, desde su conceptuación y diseño a su prestación y seguimiento, pasando por su elaboración/desarrollo.

Como síntesis de lo anterior, recogemos las palabras de Galgano (1993, p. 44), encuadradas en el marco del modelo de la $\mathrm{CWQC}^{6}$ :

"Si es verdad que la calidad es el factor predominante, tengo que conseguir que todas las personas de la empresa se interesen por ella, y lo que es más, si la calidad es lo principal, ésta debe impregnar toda la empresa, lo que quiere decir que la empresa debe estructurarse en función de la calidad".

(3) Galgano mantiene que aceptar que "calidad es sinónimo de coste" es una "grave limitación para la mejora de la calidad, ya que sentimos que haciendo las cosas de esta manera soportaremos mayores costes". Vid. GALGANO, A. (1993): Calidad Total, Madrid, Díaz de Santos, p. 23.

(4) En el mismo sentido se mueven los planteamientos de la Company Wide Quality Control (CWQC), que considera la mejora continua como una de las seis premisas de base, como los cimientos en que se asienta el edificio del CWQC (Vid. Galgano, Op. cit., p. 57).

(5) Galgano (op. cit., p. 32) incluye en el concepto aspectos tales como competitividad, entregas, costes, excelencia, moral, productividad, calidad del producto, cantidad, seguridad, servicio...

(6) Company-Wide Quality Control (VWQC). 


\subsection{ENFOQUES DEL TÉRMINO CALIDAD}

A nuestro juicio, se podría hablar de tres grandes enfoques del término: dos de ellos, el relativo y el absoluto, referidos a la toma o no en consideración de las metas, aspecto éste en el que, a mi juicio, radica la esencia de la calidad; el tercero, el integrado, como una visión más profunda y completa. Más profunda, porque recoge nuevos aspectos, entre los que podemos destacar las aportaciones de los movimientos de calidad; más completo, en la medida en que incorpora las aportaciones de los dos primeros.

\section{UNA PROPUESTA DE CALIDAD DE LA EDUCACIÓN. UNIVERSITARIA}

La problemática anteriormente reseñada parece poner de relieve, de una parte la necesidad de diseñar una propuesta de calidad susceptible de ser aplicable a diversas concepciones educativas de la educación universitaria y, de otra, de acudir a criterios de tipo formal que hagan posible la constatación de los puntos débiles y fuertes de los sistemas, más allá de las discrepancias ideológicas que puedan darse en cuestiones de contenido.

En efecto: mientras se trate de analizar la calidad de la educación impartida en una determinada y concreta institución, no habrá problemas en abordar la calidad entendida en sentido "absoluto", ya que será posible tomar como referencia una determinada concepción de la educación universitaria: aquella que ha sido definida por su personal, o en los ordenamientos jurídicos, o por la institución que ostenta la titularidad de la misma...

Sin embargo, cuando se deba evaluar la calidad de la educación de un determinado sistema universitario, en cuyo caso la decisión afecta a las instituciones diferentes integradas en el mismo, caben discrepancias entre unas y otras tanto en lo relativo a sus propias metas cuanto a su prioridad, por lo que las comparaciones no siempre son posibles y hasta puede ser contraproducentes al promover una determinada rigidez y homogeneidad. Es más, en ocasiones, pueden orientar la actividad y las energías de la institución hacia el logro de resultados cuantitativos sin atención a su dimensión cualitativa (más licenciados, más aprobados, más libros publicados...). En este caso, criterios como la coherencia interna, la armonía entre las partes, la eficiencia en el uso de los recursos... re'sultan especialmente adecuados.

Debo advertir de inmediato que las dos situaciones anteriores podrían ser los extremos de un continuo. Las diferencias entre instituciones integradas en un sistema no pueden, ni deben, impedir determinados elementos comunes, por lo que 
siempre cabrá la comparación en los niveles de eficiencia / eficacia en el logro de los mismos. Por ejemplo: por encima de las discrepancias de concepción y de prioridades entre dos universidades, ambas deben atender a la enseñanza y a la investigación, lo que permite obtener índices entre alumnos de doctorado inscritos y tesis defendidas, entre matriculados, examinados y aprobados... Lo que siempre será un desafío lejos de resolverse es la relación entre tales índices cuantitativos y su valor cualitativo: en ocasiones, unas pocas tesis doctorales pueden valer mucho más que un elevado número de este tipo de trabajos. Esta afirmación puede aplicarse también para comparar Facultades, Escuelas o Departamentos.

El modelo propuesto está integrado por tres grandes dimensiones, cada una de las cuales incorpora una serie de componentes, susceptibles de ser evaluados, unas y otros, mediante una serie reducida de criterios, para cada uno de los cuales habrá que contar con los correspondientes datos o evidencias.

\subsection{DIMENSIONES Y SUS COMPONENTES}

Tres son las dimensiones fundamentales de la propuesta: la concepción educativa, los medios para su logro y los resultados a conseguir. Estos dos últimos componentes figuran en el modelo europeo de gestión de la calidad (EFQM) como agentes o facilitadores, el primero, y como resultados o productos, el segundo.

En la medida en que las propuestas de calidad conceden una especial importancia a la evaluación, sea como medio para comprobar y controlar, sea como instrumento para el diagnóstico, identificación de puntos fuertes y débiles y las consiguientes decisiones orientadas a la mejora, la propuesta debe admitir una nueva dimensión: el contexto de la institución, tanto en el sentido del marco social y ambiental en que se encuentra enclavada cuando en lo relativo a las circunstancias que la condicionan: antecedentes, disponibilidades, formación, conflictos no resueltos, así como a las características que la define, como puede ser, en nuestro caso, la especificidad del modelo educativo. Esta nueva dimensión facilita las valoraciones en que se concreta la evaluación.

\subsubsection{El contexto}

Calidad y evaluación de la calidad son dos realidades íntimamente relacionadas; si no tiene sentido la segunda sin estar orientada a la primera, no cabe pensar en alcanzar la primera sin contar con un adecuado sistema de evaluación de la misma. 
Ahora bien, toda evaluación necesita de criterios y de referencias. Los criterios son normas para juzgar las cosas (una redacción, un trabajo, el planteamiento de un problema...); las referencias ponen esa misma realidad en relación con otra que se toma como elemento de comparación.

La valoración de la calidad de la educación de una organización educativa no deberá realizarse sin tomar en consideración, de alguna manera, aspectos tales como su nacimiento y evolución, las finalidades a las que sirve, sus peculiaridades organizativas, sus destinatarios, los recursos disponibles...

Un ejemplo patente lo tenemos en las instituciones educativas a distancia: su juventud; su espectacular desarrollo, por cierto, claramente desequilibrado entre servicios a prestar y medios y recursos disponibles; el perfil peculiar de sus destinatarios; la especificidad de su metodología... son elementos contextuales que deben ser tomados en consideración cuando se valore la calidad de la educación impartida y se tomen las decisiones que deriven de tal evaluación.

\subsubsection{Concepción educativa}

En esta dimensión de la calidad, básica para nuestra propuesta por ser condicionante de las dos siguientes, podemos distinguir tres grandes componentes:

- Los aprendizajes académicos.

Da formación intelectual.

La formación integral.

Dos son las formas básicas de entender la Educación universitaria: la formación estrictamente académica y la formación universitaria integral. La primera, a su vez, se plantea a dos grandes niveles: el que se orienta al logro de aprendizajes puramente académicos, concretados en los saberes transmitidos y promovidos por el profesorado, y el más ambicioso, que busca, a través y a partir del anterior, mediante los enfoques adecuados, capacitar al alumnado para metas más ricas y profundas, como la independencia para el aprendizaje futuro, la autonomía intelectual, el descubrimiento de nuevos saberes, la innovación o el aprendizaje creativo.

Uno y otro enfoque encuentran la línea de demarcación en los objetivos que se marca el profesor en la enseñanza, a lograr por el alumnado por medio del aprendizaje: los de puro conocer, comprender, aplicar..., o los más ambiciosos, ligados al pensamiento crítico, alcanzable a través del cultivo sistemático del análisis, la síntesis, la reflexión o la misma evaluación. En esta línea van las recomendaciones del Club de Roma (Botkin, 1979), que reclama aprendizajes 
orientados a la innovación y a la creatividad, demandados por un mundo en cambio, por un saber en continua y acelerada renovación y por la necesidad de encontrar soluciones nuevas, avanzadas, a problemas cada vez más complejos.

Por su parte, la formación universitaria integral se plantea metas todavía más ambiciosas que, eso sí, se sirven como basamento del nivel más elevado de los dos anteriores. Las metas aludidas tienen como referencia la persona del universitario y no sólo el universitario como estudiante ni siquiera como futuro profesional.

En esencia, esta concepción más ambiciosa tiene que ver con la formación de personas plenas, emocionalmente competentes -según la terminología de D. Goleman-, socialmente abiertas y moralmente autónomos. De hecho, la calidad desde este punto de vista se concretaría en el logro de la autonomía moral, esto es, en la capacitación del universitario para ser dueño de sus actos, para formular, y llevar a cabo, proyectos personales de vida valiosos. Obviamente, esta capacitación encuentra en la autonomía intelectual las herramientas básicas: capacidad de pensar y criterios adecuados para valorar,... pero necesita, además, estrategias idóneas para decidir, ocasiones para hacer realidad las decisiones, hábitos para hacer vida de las opciones personales, perseverancia y fortaleza para mantener el rumbo personalmente elegido.

Si los dos primeros aspectos tienen que ver con la formación académica, de alta calidad en el segundo de ellos, el último transciende los ámbitos limitados de las aulas para abarcar la vida universitaria toda, más allá de clases, seminarios y despachos. Su cultivo, y su logro, necesita de actuaciones sistemáticas que, aunque no cuenten con la continuidad que caracteriza a las actuaciones propias de la enseñanza / aprendizaje académicos, no deberían quedar en acciones aisladas: las autoridades académicas, y el propio alumnado debidamente organizado -dada la madurez de su nivel universitario- deberían planificar y programar actividades orientadas al logro de estas metas a lo largo de toda la permanencia del alumno en la Universidad.

Interesa resaltar, no obstante, la íntima relación existente entre una sólida formación intelectual y el desarrollo personal pleno: no cabe pensar en lo segundo como una alternativa, en cierto modo poco compatible con la primera. Sí es posible, por el contrario, personas sólidamente formadas en lo intelectual y, desde luego, en el saber académico, que no alcancen una razonable plenitud y madurez humana.

\subsubsection{Los medios}

Ponerse de acuerdo en la naturaleza de la Educación es tan difícil como necesario; de hecho, las investigaciones sobre eficacia coinciden en señalar la ne- 
cesidad de que el profesorado esté de acuerdo en los objetivos básicos del aprendizaje para su logro efectivo.

Sin embargo, tal acuerdo no da lugar, necesariamente, a la deseada eficacia. Es la adecuación de lo que, genéricamente, denominamos medios y su eficiente utilización, lo que da como resultado niveles más o menos elevados de logro o de resultados.

Ahora bien, los medios son muy diferentes según los objetivos a los que sirven; pensemos en las diferencias de medios a poner cuando de lo que se trata es de que el alumnado alcance determinadas metas de aprendizaje en una asignatura, de que logre una sólida formación en la carrera elegida o de que un equipo de investigación sea eficaz o de que la universidad funcione adecuadamente para que sus servicios contribuyan a logro de las funciones que le atribuyen sus Estatutos.

En el marco de los medios son variadas las concepciones existentes; una de las más modernas, nacida expresamente de los movimientos de calidad, se encuentra en el aludido modelo de la EFQM, donde aparecen cinco grandes factores globalmente calificados de agentes o de facilitadores ${ }^{7}$. Dado que en otras aportaciones a este mismo número de RIED se estudia el modelo con suficiente detalle, nos limitamos a definir estos facilitadores de la calidad:

Diderazgo, entendido como el comportamiento y la actuación de los dirigentes y de todos los responsables, encaminado a conducir el centro hacia la gestión de calidad.

Dolítica ${ }^{8}$ y estrategia, que se refiere a la misión, valores y dirección estratégica, así como al modo en que se implantan los proyectos de la organización.

- Gestión del personal, centrado el la forma en que la institución utiliza al máximo el potencial de su personal para lograr una mejora continua.

Recursos, referido a la gestión, uso y conservación de los medios materiales al servicio de las funciones de la organización.

Drocesos: criterio centrado en la forma en que se identifican, gestionan, revisan y mejoran los procesos de las actividades del centro educativo.

(7) Si el modelo europeo se orienta a la mejora continua, el de las normas ISO tienen como orientación la certificación de calidad.

(8) En una reciente publicación del Ministerio de Educación y Cultura se utiliza el término planificación en lugar de política. Vid. Modelo Europeo de Gestión de Calidad, 1977, Madrid: Ministerio de Educación y Cultura. 
Obviamente, caben otras concepciones, más o menos amplias y desarrolladas sobre los medios; la propuesta aquí recogida, aunque no ha sido contrastada por medio de la investigación sí cuenta con el respaldo que da la experiencia de los líderes de las principales empresas europeas. Sin embargo, el modelo puede ser objeto de discrepancia, sobre todo en la medida en que la EFQM pretende su aplicación sin diferencias a los diversos ámbitos: productos y servicios.

En cuanto a la conceptuación de los factores, se cuenta con aportaciones que tienen un importante valor de cara a la toma de decisiones, si bien con la debida prudencia dado que las organizaciones educativas presentan peculiaridades que no pueden ni deben ser obviadas (la Universidad como organización funcionarial, con un elevado nivel de autonomía a la vez que dependiente en muchos aspectos, en gran medida, de los poderes públicos).

A los efectos prácticos, no obstante, se cuenta con definiciones técnicas e, incluso, con una cierta operativización de los aludidos componentes, lo que permite acudir a los mismos en los procesos evaluativos orientados a la mejora.

\subsubsection{Los resultados}

Tradicionalmente, la calidad ha venido siendo entendida como eficacia y, por tanto, como una cuestión de resultados. Sin embargo, las cosas no deberían ser así de sencillas, al menos por dos importantes tipos de razones:

- Porque la eficacia puede llegar a ser un elemento distorsionador, capaz de producir una serie de efectos no planeados ni deseados que, a la larga, puede volverse contra la organización.

Tal puede ser el caso de organizaciones que "paguen" altos precios en términos no ya puramente económicos sino de relaciones humanas (tensiones entre el personal, esfuerzos excesivos y desproporcionados, hipermotivación...) con sus consecuencias para etapas posteriores.

- Porque, en la medida en que se planteen reductivamente los resultados, algo habitual por otra parte en las organizaciones educativas, puede lograrse la eficacia a costa de los objetivos más elevados de la propia organización. Así se manifiestan Ivor Morrish y Lawrance Stenhouse. El primero mantiene que se puede "ganar eficacia y economía en el campo de la educación a expensas del desarrollo del pensamiento reflexivo, de las actitudes sociales y de la creatividad" (Morrish, 1978, p. 193). Stenhouse (1984, p. 164) por su parte afirma que 'El mejor modo de asegurar el 'éxito' es mantener un bajo nivel de aspiración"; así, los profesores que se proponen metas poco elevadas o niveles bajos de logro en metas elevadas podrán lograr resultados aparentemente superiores. 
Parece evidente, por tanto, que los resultados deben cumplir dos grandes requisitos: abarcar todos y cada uno de los componentes de calidad de la segunda dimensión de nuestra propuesta, la concepción educativa, con la debida ponderación y relación', y, según el propio modelo de calidad de la EFQM, plantearse otros factores colaterales, como son los de satisfacción, tanto del personal cuanto de la clientela, o el propio impacto de la organización en el marco social en que se encuentra enclavada.

\section{NIVELES DE ACTUACIÓN}

Una de las cuestiones que se nos ha planteado a lo largo del punto anterior tiene que ver con los diferentes niveles a que se mueve una organización de cierta complejidad, como es el caso de una Universidad. Dado que cada nivel tiene sus propias funciones y se orienta al logro de resultados específicos, necesita de diferentes medios para su logro.

A nuestro juicio, una Universidad presenta, al menos, cuatro niveles diferentes, cada uno con sus propias responsabilidades. A ello conviene añadir que, en ocasiones, se dan notables superposiciones de actuación y que no siempre las funciones están debidamente asignadas. Es más, que, probablemente, entre la legislación al respecto y las recomendaciones técnicas de la calidad, puede no darse la suficiente congruencia de planteamientos.

\subsection{PRIMER NIVEL: GOBIERNO DE LA UNIVERSIDAD}

A la Universidad como tal, a través de sus Órganos representativos, le corresponde, en primer lugar, fijar lo que, en términos de los movimientos de calidad, se denomina la misión. La misión debe entenderse como un elemento aglutinador de todas las actividades y decisiones universitarias, como un criterio para establecer prioridades, como un punto de referencia a la hora de valorar alternativas.

Obviamente, el establecimiento de la misión requiere un elevado consenso, razón por la cual el Órgano adecuado para su definición es el Claustro y, si se me permite, la comunidad universitaria toda, a través de un referéndum al respecto ${ }^{10}$.

(9) El lector puede encontrar un análisis de la multidimensionalidad de los resultados en las aportaciones de RODRÍGUEZ ESPINAR, S. Modelos de investigación sobre el rendimiento académico. Problemática y tendencias, y DE LA ORDEN, A. Hacia una conceptualización del rendimiento educativo, ambas en Revista de Investigación Educativa, 3, (6), 284-304 y 271-283 respectivamente.

(10) La Carta Magna de las Universidades Europeas (Bolonia, 1988) establece algo que podría considerarse su misión: "...una comunidad académica que, de modo riguroso y crítico, contribuye a la tutela y desarrollo de la dignidad humana y de la herencia cultural mediante la investigación, la enseñanza y los diversos servicios ofrecidos a las comunidades locales, nacionales e internacionales". 
El Claustro, como representación máxima de la Universidad, debería promover y estimular la participación de todo el personal, con la vista puesta en su implicación y subsiguiente compromiso con las metas de mejora sistemática.

$\mathrm{Al}$ Equipo de Gobierno, en la medida en que el Rector cuenta con el respaldo de una mayoría del Claustro, le corresponde, además de actualizar y mantener viva la misión, liderar las actuaciones que estimulan, promueven, mantienen y mejoran la calidad. Ni que decir tiene que todas estas actuaciones deben ser coherentes con la misión y que, para ser eficaces, deben contar con el respaldo de la Comunidad universitaria a través de Órganos representativos, como es la Junta de Gobierno.

En la medida en que lo que interesa es no tanto la aprobación de propuestas de mejora cuanto su efectiva realización, el Equipo debe hacer gala de un estilo de actuación caracterizado por el diálogo, la participación y la búsqueda de apoyo y de compromiso de la Comunidad. El compromiso suele exigir muchas actuaciones orientadas al convencimiento de la bondad de las decisiones, a tomar o ya tomadas; en ocasiones, tales actuaciones son de índole colectiva, pero pueden resultar idóneas, y eficaces, aquellas otras de tipo individual que buscan el apoyo de personas líderes capaces de arrastrar a grupos significativos del personal.

Ello no obstante, cuando determinados miembros de la comunidad se automarginan o, todavía peor, incumplen las normas así alcanzadas, al Equipo de Gobierno le corresponde la tarea de hacer cumplir los acuerdos, incluyendo en este planteamiento las acciones disciplinarias pertinentes.

Entre las actuaciones concretas que podríamos reseñar, encaminadas a la calidad a nivel de la organización toda, podemos destacar las siguientes:

\subsubsection{Conocer la situación de la Universidad}

$\mathrm{Si}$, como suele afirmarse, la información es poder, en este caso la información es la base para la toma de decisiones acertadas.

La utilización de consultas de diversa naturaleza, alcance y metodología, permite llegar a un diagnóstico, base para determinadas actuaciones que, según los datos recogidos, pueden contar con un mayor o menor grado de respaldo.

No debería entenderse esta afirmàción en el sentido de que toda decisión deba contar con el apoyo inicial de la mayoría; en ocasiones, las decisiones orientadas a la calidad pueden ser impopulares, pero, para que lleguen a ser aceptadas, siempre será necesario contar con una información válida sobre la situación de partida. 


\subsubsection{Promover la participación}

Una organización pública como es la Universidad, de carácter notablemente funcionarial, con personal altamente cualificado, estructurada en órganos dotados de notable grado de autonomía -caso de los Departamentos-, no puede funcionar sobre la base de normas impuestas. Es, por el contrario, conveniente y necesario el acuerdo en su bondad y adecuación para que sea posible el libre compromiso con las mismas.

A tal acuerdo se puede llegar, tras haber logrado un fuerte respaldo a la $m i$ sión de la Universidad, por la doble vía de la transparencia en la información y de los canales abiertos a la participación, unos de carácter natural, espontáneo, mediante facilidades para la comunicación de doble vía, y otros de naturaleza representativa, mediante debates para la toma de acuerdos de relieve, debates que pueden ir más allá de los obligados en los Órganos de gobierno.

Para ello, es necesario crear un clima participativo cuyo primer paso es la sensibilización del personal hacia los problemas de la Universidad y su implicación en la solución de los mismos. Debe huirse de la indiferencia, del desconocimiento y del aislamiento que deja todo en manos de quienes en cada momento gobiernan.

\subsubsection{Estimular las relaciones humanas}

Las organizaciones son, antes que otra cosa, conjuntos de personas teóricamente unidas por unos objetivos en cuyo marco han de encontrar la oportunidad de llevar adelante su desarrollo personal y profesional.

Con frecuencia, las tensiones entre el personal son una de las causas fundamentales de ineficiencia e ineficacia de las organizaciones. En consecuencia, crear las condiciones para que las relaciones humanas, no exentas de un razonable nivel de conflicto y tensión, permitan concentrar el esfuerzo y el trabajo en los objetivos de la organización, es una de las funciones fundamentales de todos aquellos que ostentan determinados cargos, desde el de Rector al de Director de Departamento, pasando por Vicerrectores y Decanos.

\subsubsection{Asignar responsabilidades}

La gestión de la Universidad necesita de la participación de muchos de sus miembros en puestos de responsabilidad a diferentes niveles.

La traducción operativa de las líneas de actuación marcadas por los Equipos de Gobierno encuentran en este punto una parte importante de su eficacia. Por tanto, la selección de las personas que han de ejercer tales funciones es una de las 
tareas más delicadas que deben ser cuidadas por quienes gobiernan la universidad y de cuyo éxito depende en gran medida su propia eficacia.

\subsubsection{Allegar, repartir adecuadamente y optimizar los recursos disponibles}

Poco a poco se avanza en la dirección de una co-financiación de las universidades. De la dependencia total con respecto a las autoridades políticas se está pasando a la financiación mixta. En ella las universidades deben encontrar fuentes que les permitan ir más allá de una mortecina supervivencia.

\subsubsection{La búsqueda de recursos}

La búsqueda de fuentes de financiación va a encontrar en las tasas de matriculación uno de los componentes fundamentales, lo que representa la necesidad de buscar la satisfacción del alumnado y su fidelización a la universidad a través tanto de la debida atención personal y académica cuanto de la excelencia de la formación ofrecida.

Junto a ello, será preciso encontrar fuentes diferentes que, eso sí, deben resultar compatibles con la misión de la Universidad: la financiación no debe lograrse aumentando los recursos mediante decisiones eficaces a corto plazo, sino a través de una oferta de calidad. De no ser así, los éxitos económicos de hoy pueden devenir en fracasos académicos y económicos el día de mañana.

\subsubsection{Reparto de los recursos}

En el reparto de los recursos es preciso contar con criterios, debidamente establecidos, públicos y objetivamente aplicados. Los criterios, obviamente, deben estar en sintonía con la misión: los recursos son medios para lograr los objetivos y se convierten en herramientas al servicio de las prioridades enunciadas y aprobadas.

\subsubsection{Optimización de los recursos}

Una última cuestión tiene que ver con la optimización de los recursos. Con frecuencia, los recursos disponibles están seriamente infrautilizados, pero la inversión realizada en ellos limita la disponibilidad de medios en otros campos tal vez tan necesarios e importantes como aquéllos. La debida relación y adecuación entre inversión, uso y utilidad debería ser adecuadamente valorada. 
En otras ocasiones, la ineficiencia de los recursos radica en la incorrecta definición de los procesos necesarios para que el trabajo sea más ágil y rentable. El estudio de los procesos es un punto clave en cualquier política de calidad: hacer bien las cosas a la primera, en el menor tiempo posible y con la mayor satisfacción de quienes lo hacen.

\subsubsection{Evaluar sistemáticamente la situación como base de decisiones de mejora} continua

Probablemente no exista ninguna estrategia potencialmente más eficaz para la mejora continua que la existencia de un plan sistemático de evaluación de las organizaciones.

La información que se genera, sobre la base de la actitud de autocrítica y de disposición al cambio que implica todo proceso evaluativo, pone a los responsables ante sus puntos fuertes y débiles, facilitando el acierto en las decisiones a tomar. Como muy bien dijo Lafourcade hace ya muchos años (1969, p. 16):

"Sin la indicación de objetivos, el proceso sería un barco a la deriva; sin un buen complejo metodológico, una acción insegura y azarosa; sin aprendizaje, un esfuerzo inútil; sin evaluación, una empresa de la cual se desconocería su eficiencia, pese a que la tuviera; $y$ sin reajuste, una tarea a medias"

Debe quedar claro, no obstante, que la evaluación no produce per se los efectos beneficiosos; es su utilización adecuada, la implicación del personal y las consecuencias derivadas -toma de decisiones-, sobre la base de una información fiable, válida y valiosa, lo que la convierte en esa potente herramienta a que nos referimos.

\subsubsection{Estimular las actividades de formación integral de los alumnos}

La propuesta de calidad que venimos desarrollando concede una especial importancia a la formación integral de los alumnos, algo que, dada su edad, debe gozar tanto de un carácter sistemático en su organización cuanto de voluntariedad en la participación.

La creación de oportunidades, la facilitación de recursos y apoyos, el estímulo a la participación del profesorado y la corresponsabilización de las asociaciones de estudiantes son las fórmulas idóneas de actuación. 


\subsubsection{Diseñar una política general de personal}

Las organizaciones son lo que sea su personal, pero un mismo personal, adecuadamente estimulado, organizado y reconocido puede ofrecer resultados notablemente más elevados.

Las universidades deben solucionar un importante problema: el relativo a la coordinación de funcionamiento entre el personal docente y el de administración y servicios, implicados ambos en la meta final de la calidad.

En la política general de personal, al Equipo de Gobierno le incumbe, ante todo, y sobre la base de la participación a que ya nos hemos referido:

La definición y asignación de funciones, sobre la base de la capacitación acreditada por cada uno.

La creación de oportunidades de formación y actualización permanente, tanto en temas generales como específicos del puesto de trabajo.

- El diseño de un plan de desarrollo profesional, con las adecuadas posibilidades de promoción y mejora.

- La creación de un sistema de asesoría permanente.

- La elaboración de un plan sistemático que permita conocer el grado de satisfacción existente y las demandas de mejora.

\subsection{SEGUNDO NIVEL: GESTIÓN DE LA TITULACIÓN}

La Universidad está al servicio de la formación superior de las nuevas generaciones, sea desde la perspectiva de los lideres políticos, sociales o profesionales, sea desde el liderazgo científico o ideológico.

La formación superior encuentra en las diversas titulaciones universitarias el elemento aglutinador. Una titulación reúne, organiza, estructura y desarrolla los esfuerzos y actuaciones de un conjunto de profesores, debidamente agrupados en Departamentos, y de un amplio elenco de otros profesionales de la administración y de los servicios.

Si bien la organización que deriva de la legislación vigente concede un extraordinario papel a los Departamentos, a nuestro juicio no son la unidad adecuada para el estudio de la titulación ya que su ámbito de actuación es el Área de conocimiento; al margen de su definición, no siempre correcta ni acertada, el Área se preocupa de sectores más o menos amplios de la formación del universitario, pero, en modo alguno, de toda la formación. 
La actividad del Departamento en el marco de las enseñanzas tiene dos importantes frentes: la coherencia interna de los planteamientos y su congruencia con el perfil de la titulación, a cuya definición debe contribuir de forma destacada.

Sin embargo, la Titulación debe encontrar alguna forma de funcionamiento que garantice que el perfil de la misma es abordado en su totalidad, sin superposiciones ni lagunas, y que la forma de hacerlo resulta coherente entre las aportaciones de las diversas Áreas de conocimiento. La figura adecuada para las grandes decisiones es la Junta de Facultad; sin embargo, el día a día debe ser la tarea fundamental de los Equipos decanales, solos o en compañía -según los casos- de los Directores de los Departamentos.

La gestión de la Titulación es, sin duda, una función clave de los Decanatos; en ella deben evidenciar liderazgo, capacidad de coordinación y adecuada organización, y todo ello estando al día de las opiniones del personal, difundiendo la información que reciben y generan, promoviendo la participación de todos los estamentos en cuanto tales y del personal a título individual, favoreciendo el clima de cordialidad en las relaciones y agilizando las respuestas a las demandas, las soluciones a los conflictos y la asimilación de las frustraciones que puedan producirse.

\subsection{TERCER NIVEL: ORGANIZACIÓN DE LAS ENSEÑANZAS}

La calidad exige idoneidad de respuestas a las diferentes demandas. En el campo de las enseñanzas, la principal de estas cuestiones radica en la selección de las personas encargadas de dar tales respuestas al alumnado. Junto a ello, la asignación de funciones a las mismas y la coordinación de actuaciones entre los responsables.

Pues bien, son los Departamentos los Órganos clave en los procesos de búsqueda de candidatos, de formación de aspirantes, de selección de los más idóneos, de asignación de responsabilidades, de promoción a niveles superiores, de reparto de la carga docente, de estímulo y de facilitación del desarrollo profesional...

\subsubsection{Selección, formación y promoción}

La selección y la promoción del profesorado son en la actualidad dos problemas de envergadura en la vida académica de la Universidad. En el marco del primero de ellos, es preciso buscar el equilibrio entre la rentabilización de las inversiones en formación que lleva a cabo la Universidad en sus becarios y Ayudantes y la igualdad de oportunidades. 
$\mathrm{Si}$, de una parte, es lícito, y resulta conveniente y hasta deseable, que la Universidad se haga con los servicios de las personas que forma, no lo es menos que estas personas deben hacerse merecedoras de su estabilización profesional compitiendo con personas ajenas a la misma en mérito y en capacidad. Es la única forma de que la Universidad no se haga provinciana, tenga altura de miras y huya de la indeseable endogamia.

La formación de becarios y ayudantes debería ser una de las funciones de mayor importancia de los Departamentos. De hecho, debería contar con plan sistemático de actuación tanto para las tareas investigadoras cuanto para las docentes, además de integrar planes activos para el logro de méritos que faciliten el acceso a las categorías de profesorado permanente.

La promoción es otro importante foco de problemas para la calidad. Por lo general, las posibilidades de promoción son limitadas en órganos de reducida plantilla, por lo que las tensiones entre el personal son fuertes y, si no se remedia, se convierten en fuentes de "fidelidades" y de enemistadas que pueden perdurar durante largos períodos de tiempo, incidiendo negativamente, tanto unas como otras, sobre la calidad del servicio académico.

Dos vías se atisban para minimizar estos problemas: la definición, con mucha antelación, de los campos de expansión de los Departamentos, de forma que cada profesor pueda hacerse su propia composición de lugar y planificar adecuadamente su currículo profesional, y la igualdad de oportunidades para con todo el personal que aspire a la promoción. A partir de aquí, los principios objetivos de mérito y capacidad deben primar sobre cualquier otra forma de actuación.

\subsubsection{Asignación de responsabilidades}

En lo relativo a la asignación de responsabilidades, debería acudirse a criterios objetivos de mérito y capacidad y, en los casos en que varios profesores deban compartir sus tareas, a una colaboración en que se garantice la participación de cada miembro del equipo según sus derechos, formación y antigüedad.

\subsubsection{Coordinación}

La coordinación de planteamientos es algo deseable en una política de calidad, e imprescindible cuando los grupos comparten responsabilidades sobre el mismo grupo de alumnos; se trata, en definitiva, de ofrecer al alumnado un mensaje coherente, mensaje que debe derivar de reuniones técnicas en las que el profesorado pueda debatir sus planteamientos con sus colegas. 


\subsubsection{Funcionamiento}

Los Departamentos, en gran medida, son el resultado de decisiones con un elevado nivel de autonomía. Si la corrección -rigor, validez de los criterios...- con que se tomen contribuye al grado de calidad de las enseñanzas, el modo en que se llevan a cabo ayuda a la conformación de lo que hemos denominado clima, en el que las relaciones humanas favorecen o entorpecen y hasta obstaculizan el buen desarrollo de la labor universitaria.

Un elemento clave para la eficiencia de los Departamentos radica en el liderazgo de sus dirigentes; su prestigio profesional, su buen hacer personal y su actitud de servicio contribuyen sobremanera al clima sosegado que deviene en favor de la calidad académica. Junto a ello, se debe destacar la importancia del desarrollo democrático de la vida departamental y su desarrollo a través de la existencia de criterios previos, públicos y objetivables, que faciliten decisiones objetivas correctas y no actuaciones "ad personam", evitando la creación de situaciones crispadas, con reflejo e incidencia en la vida académica y en la calidad del servicio.

\subsection{CUARTO NIVEL: DESARROLLO DE LAS ENSEÑANZAS}

La relación directa profesor-alumno tiene en los equipos docentes la concreción necesaria. Si en los movimientos de calidad, en el ámbito de los servicios, un punto clave es, justamente, el de la relación directa entre la organización prestataria y el receptor del servicio, no cabe duda de que uno de los elementos clave de esta relación es el profesor, o el conjunto de varios profesores cuando un mismo servicio -una asignatura- es impartida, dado su tamaño, por más de uno.

Cinco son aquí las cuestiones clave en el marco de la calidad:

- Los programas en los que toman cuerpo las asignaturas, dando al término programa su más profunda acepción: plan para hacer de un temario una realidad aprendible"l por el alumnado.

$\mathrm{Al}$ programa en cuanto temario hay que pedirle rigor y altura universitarios: actualización, estructuración, cientificidad, respuesta a las demandas sociales y profesionales en su caso.

En cuanto plan para hacer del temario una realidad aprendible, le es exigible la incorporación de cuantos elementos motivadores, estimula-

(11) Obviamente, por coherencia con lo dicho al hablar de la concepción educativa de la educación, el concepto de aprendizaje se toma aquí en su acepción más rica y profunda. 
dores y facilitadores aconseja la psicología del aprendizaje y la didáctica. Un elemento clave es el de una cierta personalización, tanto en los objetivos y motivación cuanto en la metodología y en la misma evaluación (niveles diferenciados) ya que si, de una parte, no todos los contenidos del temario tienen la misma relevancia científica o técnica o la misma importancia y demanda social, de otra, las capacidades, intereses, motivación y capacidad de aprender del alumnado presentan una gran dispersión, como han puesto de relieve todas las investigaciones psicopedagógicas al respecto.

- La atención durante el proceso de aprendizaje. La enseñanza, con frecuencia, es una realidad que el profesor puede programar y desarrollar en la forma prevista. Sin embargo, el aprendizaje es una tarea profundamente personal en la que es fácil que se evidencien dificultades, bien deriven de carencias en el sujeto que aprende (ausencia de conocimientos previos o prerrequisitos, limitaciones en capacidad, falta de motivación), bien en la propia enseñanza (exposiciones obscuras, textos inadecuados, lenguaje inasequible...), bien en el propio contexto del estudiante (problemas familiares, profesionales y personales).

La atención durante el proceso representa la forma más rica de personalización: el profesor ayuda al alumno concreto que experimenta dificultades o que demanda orientaciones para superarse. Desde esta perspectiva, el cumplimiento de los horarios es un elemento clave de la calidad; de hecho, va mucho más allá del mero cumplimiento para convertirse en elemento nuclear, y no sólo en cuestiones reactivas -respuesta a consultas- sino anticipadoras -orientaciones continuadas al estudio, recomendación de actividades...

- La forma de atender. La calidad exige calidez en el trato, disponibilidad, amabilidad..., además de agilidad y efectividad en las respuestas. Con frecuencia, la distancia y frialdad en el trato, producen, intencionadamente o no, la reducción de consultas al profesorado.

Por contra, la actitud abierta de éste, su estímulo a solicitar ayudas, su respuesta ágil, clara y amable, animan al alumno a seguir utilizando de los servicios del profesor, con la consiguiente mejora de su aprendizaje ${ }^{12}$.

- La evaluación formativa o para la mejora. Las pruebas formativas, llevadas a cabo durante el proceso de aprendizaje, permiten al alumno y al

(12) En una investigación dirigida por el autor, en que se contrastaba un modelo de evaluación formativa -orientada a la mejora- frente a otro sumativo -limitado al control y la calificación- en el aprendizaje de las Matemática a nivel de primer curso de ciplomatura, la actitud del profesor condujo a una media de 9 preguntas por clase en el primer caso frente a otra de 1,5 en el segundo. 
profesor tomar conciencia de los progresos, de los errores y de las lagunas en el aprender, con la consiguiente posibilidad de tomar a tiempo las decisiones pertinentes que se traducirán err mayores niveles de éxito al realizar las pruebas.

Con mentalidad formativa cabe, incluso, una evaluación inicial, previa al proceso de aprendizaje, orientada a corregir las carencias en aspectos que puedan considerarse pre-rrequisitos para el éxito.

- La mejora del programa. Por mucho cuidado que se ponga en la elaboración del programa, dada diversidad de circunstancias ambientales y personales en que éste llega a su destinatario es previsible que no responda plenamente a la calidad deseada. De hecho, la calidad es siempre una cuestión de mejora continua.

La atención durante el proceso de aprendizaje a que ya nos hemos referido no sólo sirve al alumno que aprende y va superando sus concretas dificultades; las dificultades y las consultas ya dan al equipo docente una serie de pistas de los aspectos del programa que deben ser objeto de mejora.

Pero, junto a ello, la pruebas -tanto formativas como sumativas- $\mathrm{y}$ las actuaciones específicas -evaluaciones del propio profesorado, de su metodología, de su atención, de sus materiales...- le proporcionan una importante información que, si es debidamente utilizada, le permiten la toma sistemática de decisiones de mejora.

Instrumentos de los sistemas de calidad, como el diagrama de Paretto, el histograma o el diagrama de causa / efecto, pueden ayudar a los equipos docentes en el proceso de mejora continua de su programa.

\section{LAS PECULIARIDADES DE LOS SISTEMAS A DISTANCIA}

Las universidades que siguen los sistemas a distancia no deben diferenciarse del resto más allá de su específica metodología, palabra que abarca un conjunto de elementos materiales, funcionales e instrumentales orientados a facilitar el aprendizaje realizado por personas que, salvo contadas excepciones, no mantienen una relación personal presencial y simultánea con sus profesores.

La relación mediada entre alumnos y profesores y el perfil diferencial de éstos son los elementos que justifican la peculiaridad de la metodología que, por otra parte, se debe orientar a objetivos de la misma naturaleza y nivel que los del resto de universidades. 
En tal sentido, los desafíos de las Universidades a distancia se concentran en los niveles primero y cuarto de los arriba estudiados. A los responsables del primero de ellos les corresponde facilitar los medios adecuados que permitan al profesorado tener éxito en la enseñanza a distancia o mediada, tales como: asesoría y formación para la elaboración y mejora sistemática de materiales de autoaprendizaje y atención mediada, medios para este tipo de atención (teléfono, fax, videoconferencia, radio, TV, infraestructura informática), facilidades para la comunicación con los profesores tutores e información sobre el perfil diferencial de sus estudiantes.

Por su parte, el desafío de los profesores no es otro que saber estimular, promover y facilitar el aprendizaje por parte de los alumnos a través de los medios, tanto impresos como tecnológicos e informáticos, ayudarles y orientarles en su proceso de aprender y utilizar los resultados de la evaluación como medio para la mejora continua.

En los casos en que la organización de la educación a distancia incorpora, además de profesores, a profesores tutores en centros asociados, es preciso encontrar fórmulas que permitan la coordinación entre ambos tipos de profesionales y una adecuada distribución de funciones entre ellos. El desarrollo de este punto supera con mucho las posibilidades de esta colaboración ${ }^{13}$.

\section{EL PROBLEMA DE LA INVESTIGACIÓN}

Tradicionalmente se ha encomendado a la Universidad la impartición y el desarrollo del saber. Si la primera función se asocia a la enseñanza / aprendizaje, concretada en lo esencial en las Titulaciones (aunque, cada vez más, la educación permanente y la extensión universitaria va adquiriendo mayores desarrollos), la segunda tiene que ver, especialmente, con la investigación.

Si bien en la evaluación de la calidad de las universidades se incorporan tres grandes unidades: la enseñanza, la investigación y la gestión, en un documento como el presente, destinado a reflexionar sobre la calidad de la educación, su tratamiento debe centrarse no tanto en lo relativo a su naturaleza, a los factores que contribuyen a su calidad y a los criterios de eficacia, cuanto en lo que se refiere a sus relaciones con la enseñanza, el aprendizaje y la formación del alumnado.

(13) El IUED publicará en breve una propuesta formulada por el autor en el marco de las I Jornadas de la función tutorial: su pasado, su presente y su futuro, que han tenido lugar en Madrid, en noviembre de 1997. 
Así, en este contexto, tres podrían ser los aspectos fundamentales a tener en cuenta y que, por falta de espacio, no se desarrollan:

- La necesidad de equilibrio entre la dedicación a la docencia y a la investigación, de forma que el desarrollo de una función no vaya en detrimento de la otra.

- La influencia que la investigación juega sobre la calidad de la acción docente y formativa del profesor sobre el alumnado, tanto por lo que representa de actualización y avance del saber cuanto por la actitud que refleja y por su repercusión en la metodología, más activa e indagativa que meramente pasiva y receptiva.

D Su carácter de componente de la formación del alumno, orientada a su capacitación para estar al día en temas de investigación y, en su caso, para investigar en el futuro, bien en su ámbito profesional bien, precisamente, como profesional de la misma.

Dejaremos constancia, no obstante, de que la investigación en la universidad adolece de graves problemas, problemas que hacen difícil su ubicación en el marco de la calidad, mereciendo una especial atención el referente a su valoración como mérito.

La complejidad de la investigación, y su deseable eficacia, parece exigir el trabajo en equipo, incluso de tipo multidisciplinar. La constitución de equipos de investigadores va ligada a la existencia de líderes científicos, capaces de plantear problemas, diseñar proyectos, elaborar metodologías, a los que cada uno de sus miembros aporten lo mejor de su formación y de su esfuerzo.

Sin embargo, el hecho de que tal valoración se realice en términos de personas concretas, conduce a que difícilmente se puedan consolidar unos cuantos, muy pocos, equipos de investigación en la Universidad, en el seno de los Departamentos.

\section{REFERENCIAS BIBLIOGRÁFICAS}

AIDIPE. (1989). Evaluación de Centros. Nuevas perspectivas en la investigación sobre organizaciones educativas. Revista de Investigación Educativa, monográfico, 7 (13).

Álvarez-Tostado, C. (1997). Calidad de la Educación. Entre el eslogan y la utopía. Buenos Aires: Magisterio del Río de la Plata.

Amat, O. (1993). Costes de la no calidad. Barcelona: EADA Gestión. 
Aparicio Izquierdo, F. Y GonzÁlez TiRados, R.M. (1994). La calidad de la enseñanza superior y otros temas universitarios. Madrid: ICE de la Universidad Politécnica de Madrid.

Asociación Española para LA CALIDAD (AECC) (1995). Calidad por y para el Hombre. Ponencias del VI Congreso Nacional de la Calidad. Madrid: AECC-Ediciones Gestión 2000 SA.

AsSOCIATION DES UNIVERSITÉS EUROPÉENNES-CRE (1997). Seminaire de gestion et de réflexion por dirigeants universitaires, La gestion de l'Univesité: ses enjeux, ses partenaires. Bourdeaux, France.

BotkIN, J.W. y otros (1979). Aprender, horizonte sin limites (Informe del Club de Roma). Madrid: Santillana.

CECE-Club Gestión de Calidad (1997). Guia de autoevaluación. Centros Educativos o formativos no universitarios. Modelo Europeo de Calidad total en la gestión, Madrid: ITE de la CECE.

CONSEJo DE UnIVERSIDAdes (1995). Programa de Evaluación Institucional de la Calidad de las Universidades. Madrid: Secretaría General del Consejo de Universidades.

Consejo de Universidades (1995). Proyecto Piloto Europeo para evaluar la calidad en la Enseñanza Superior. Informe Europeo, Madrid.

CONSEJO DE UNIVERSIDAdes ( $\sin$ fecha). Plan Nacional de Evaluación de la Calidad de las Universidades. Madrid: Secretaría General.

DiEz Hochleitner, R. (1995). Educación y desarrollo. Aprender para el futuro. Madrid: Fundación Santillana.

Escotet, M.A. (1984). Técnicas de evaluación institucional de la Educación Superior. Madrid: MEC.

Galgano, A. (1993), Calidad Total. Madrid: Díaz de Santos.

Garcia ARETIO, L. (1994). Educación a Distancia hoy. Madrid: UNED.

Gento Palacios, S. (1996). Instituciones educativas para la calidad total. Madrid: La Muralla.

Goleman, D. (1996). Inteligencia emocional. Barcelona: Kairós.

INTERNATIONAL COUNCIL For EduCATIONAL DEVELopment (1988). La Reforma Universitaria Española. Evaluación e Informe. Madrid: Consejo de Universidades.

LAFOURCADE, P.D. (1969). Evaluación de los aprendizajes. Buenos Aires: Kapelusz.

LAFOURCADE, P.D. (1984). La evaluación en organizaciones educativas centradas en logros. México: Trillas.

LÓPEZ Rupérez. F. (1994), La gestión de Calidad en Educación. Madrid: La Muralla.

Miguel, M. DE, Ginés Mora, J. y Rodríguez, S. (edits) (1991), La evaluación de las Instituciones Universitarias. Madrid: Consejo de Universidades.

Ministerio de Educación y Cultura (1997). Modelo Europeo de Gestión de Calidad. Madrid: Centro de Publicaciones. Secretaría General Técnica.

Moltó, T. y Oroval, E. (1984). Costes y rendimientos en la Enseñanza Superior. Barcelona: ICE de la Universitat de Barcelona.

MuNoz-RePIso, M. y otros (1995). Calidad de la Educación y Eficacia de la Escuela. Madrid: CIDE. 
OCDE (1991). Escuelas y calidad de la Enseñanza. Barcelona: Paidós-MEC.

O.E.I. (1994). Evaluación de la Calidad de la Educación. Revista Iberoamericana de Educación, (monográfico), 10.

Rosander, A.C. (1994), Los catorce puntos de Deming aplicados a los servicios. Madrid: Díaz de Santos.

SCHARgel, F.P. (1997). Cómo transformar la Educación a través de la gestión de la Calidad Total. Madrid: Díaz de Santos.

Sebastian Pérez, M.A. y otros (1994). Gestión y Control de Calidad. Madrid: UNED.

SEnLle, A. (1994), Calidad y liderazgo, 20 ed. Barcelona: Gestión 2000 S.A.

SENLle, A. y Stoll, J. (1994). ISO 9000. Calidad total y normalización. Barcelona: Gestión 2000 S.A.

SENLle, A. y Vill.AR, J. (1996). ISO 9000 en Empresas de Servicios. Barcelona: Gestión 2000 S.A.

Wilson, Jh.D. (1992). Como valorar la calidad de la enseñanza. Madrid: MEC.

\section{PERFIL ACADÉMICO-PROFESIONAL DEL AUTOR}

Ramón Pérez Juste es Catedrático de Pedagogía Experimental. Ha desempeñado los cargos de Director del Departamento de Métodos de Investigación y Diagnóstico en Educación y Decano de la Facultad de Educación. En la actualidad es Vicerrector de Ordenación Académica y Profesorado de la UNED. Trabaja en temas de calidad, ligada tanto a la gestión cuanto a los proyectos educativos (educación en valores), y de evaluación, tanto del aprendizaje cuanto de la enseñanza y de las instituciones educativas.

Ramón Pérez Juste

UNED

C/ Bravo Murillo, 38

28015 Madrid (España)

Fax: +34913986038

Correo Electrónico:Rperez@bmuned.es 